\title{
Characterisation of the role and regulation of Ultrabithorax in sculpting fine-scale leg morphology
}

\author{
Alexandra D. Buffry ${ }^{1}$, Sebastian Kittelmann ${ }^{2}$ and Alistair P. McGregor ${ }^{1}$ \\ ${ }^{1}$ Department of Biological and Medical Sciences, Faculty of Health and Life Sciences, Oxford Brookes University, Oxford, OX3 0BP, \\ United Kingdom. \\ ${ }^{2}$ Centre for Functional Genomics, Department of Biological and Medical Sciences, Faculty of Health and Life Sciences, Oxford \\ Brookes University, Oxford, OX3 OBP, United Kingdom.
}

Key words: Drosophila, enhancer, evolution, development, gene regulation, Ultrabithorax, Hox genes

\begin{abstract}
Hox genes are expressed during embryogenesis and determine the regional identity of animal bodies along the antero-posterior axis. However, they also function post-embryonically to sculpt fine-scale morphology. To better understand how Hox genes are integrated into post-embryonic gene regulatory networks, we further analysed the role and regulation of Ultrabithorax (Ubx) during mesothoracic (T2) leg development in Drosophila melanogaster. Ubx represses leg trichomes in the proximal posterior region of the T2 femur (the so-called naked valley) and we found that it likely does so through activating the expression of microRNA-92a. We also identified a T2 leg enhancer of $U b x$ that recapitulates the temporal and regional activity of this Hox gene in these appendages. Analysis of motifs in this enhancer predicted that it is bound by Distal-less (DII) and we found that knockdown of DIl results in the loss of trichomes on the T2 femur. This suggests that while Ubx activates microRNA-92a to repress trichomes in the naked valley region of the proximal femur, DIl may repress Ubx more distally to enable formation of trichomes. Taken together our results provide insights into how $U b x$ is integrated into a postembryonic gene regulatory network to determine fine-scale leg morphology.
\end{abstract}

\section{Introduction}

The Hox genes encode an important and conserved family of transcription factors (TFs) that are expressed during embryogenesis to determine the identity of body regions along the anteroposterior axis of animals (Carroll et al., 2005; Hueber and Lohmann, 2008; Krumlauf, 2018; Lewis, 1978; McGinnis and Krumlauf, 1992; Pearson et al., 2005). However, Hox genes also play more subtle but important post-embryonic roles in regulating cell identify to sculpt the fine-scale morphology of structures and organs, and they have been likened to 'micromanagers' (Akam, 1998a; Akam, 1998b; Hombría and Lovegrove, 2003). Several such post-embryonic roles of Hox genes have been identified in Drosophila; for example, the specification of certain subtypes of cells in the central nervous system (Estacio-Gómez et al., 2013; Kannan et al., 2010), the regulation of the development of larval oenocytes by abdominal- $A$ ( $a b d-A$ ) (Brodu et al., 2002), and the integration of regulatory information to specify differences in prothoracic (T1) leg bristle patterning among segments and between sexes by Sex-combs reduced (Scr) (Eksi et al., 2018).

Ultrabithorax $(U b x)$ is expressed during embryogenesis and specifies the identity of thoracic and abdominal segments in Drosophila (Akam and Martinez-Arias, 1985; Castelli-Gair and Akam, 1995; White and Wilcox, 1984, 1985). Classically, this Hox gene represses wing identity and promotes haltere formation on the third thoracic (T3) segment through the direct regulation of potentially hundreds of genes (Bender et al., 1983; Diaz-de-la-Loza et al., 2020; Pavlopoulos and Akam, 2011; Tomoyasu, 2017; Weatherbee et al., 1998; White and Wilcox, 1984, 1985; White and Akam, 1985). Ubx also distinguishes the size and morphology of halteres at a more finescale level, in part through the autoregulation of differences in the expression levels between proximal and distal cells (Delker et al., 2019; Roch and Akam, 2000). In T3 legs, Ubx regulates 
the size, as well as bristle and trichome patterns in a concentration dependent manner (Casanova et al., 1985; Davis et al., 2007; Kerridge and Morata, 1982; Morata and Kerridge, 1981; Stern, 2003; Struhl, 1982). Moreover, during mesothoracic (T2) leg development, the expression levels of Ubx regulate the proximal-distal patterning of trichomes on the femur (Stern, 1998). Therefore, in addition to determining segmental identity, Ubx subsequently contributes to sculpting the finerscale morphology of several appendages.

Despite these insights into Hox gene function, we still do not fully understand how they are integrated into post-embryonic gene regulatory networks (GRNs). One approach to address this is to study the regulation of Hox genes by identifying the enhancers that are responsible for their post-embryonic expression. Indeed, several enhancers and other cis-regulatory elements of Ubx have already been identified and we are beginning to understand how they integrate information to precisely regulate the differential expression of this Hox gene to control fine-scale morphology (Bender et al., 1983; Delker et al., 2019; Irvine et al., 1993; Irvine et al., 1991; Little et al., 1990; Maeda and Karch, 2006; Magbanua et al., 2015; Müller and Bienz, 1991; Peifer and Bender, 1986; Simon et al., 1990). However, it is clear that not all Ubx enhancers have been identified and we still have much to learn about the complex regulation of this crucial gene (Davis et al., 2007; Delker et al., 2019; Magbanua et al., 2015).

Enhancers can be challenging to identify because currently there is no consensus of what genomic features mark these regions (Buffry et al., 2016; Halfon, 2019). Furthermore, although the regulatory genome can now more readily be studied with new tools such as ATAC-seq, C technologies and CRISPR/Cas9, we still don't fully understand the regulatory logic underlying enhancer function (Buffry et al., 2016; Halfon, 2019). Given their importance in development, disease and evolution, it is crucial that we continue to identify and study individual enhancers in detail, to better our general understanding of cis-regulatory regions and GRNs.

The development and patterning of trichomes among Drosophila species has proven an excellent model to study GRNs and their evolution (Arif et al., 2015; Stern and Frankel, 2013). Trichomes are short, non-sensory actin protrusions that are found on insect bodies throughout all stages of life (Arif et al., 2015). They are thought to be involved in processes such as aerodynamics, thermal regulation and larval locomotion (Balmert et al., 2011; Ditsche-Kuru et al., 2011). The larval cuticle of Drosophila displays a distinct pattern of trichomes and the underlying GRN is understood in great detail (Chanut-Delalande et al., 2006; Delon et al., 2003; Menoret et al., 2013). In brief, the gene shavenbaby (svb) appears to integrate information from upstream factors, including Ubx, and directs expression of downstream effector genes that determine the formation of the trichomes themselves (Chanut-Delalande et al., 2006; Crocker et al., 2015; Delon et al., 2003; Menoret et al., 2013; Preger-Ben Noon et al., 2016). Moreover, the convergent evolution of larval trichome patterns in different Drosophila lineages is caused by changes in enhancers of svb (Crocker et al., 2015; Frankel et al., 2011; Frankel et al., 2012; McGregor et al., 2007; Preger-Ben Noon et al., 2016; Stern and Frankel, 2013; Sucena et al., 2003; Sucena and Stern, 2000).

The T2 legs of $D$. melanogaster display a trichome pattern that is distinct from the other legs, with a patch of cuticle on the proximal posterior of the femur that is free from trichomes, known as the naked valley (NV) (Arif et al., 2013; Stern, 1998). We previously studied the GRN underlying leg trichome patterning and found that it differs in topology with respect to the larval trichome GRN (Kittelmann et al., 2018). In particular, in the developing T2 legs, the Svb-dependent activation of trichomes is blocked by microRNA-92a (miR-92a)-mediated repression of Svb target genes to generate the NV (Arif et al., 2013; Kittelmann et al., 2018). Furthermore, in contrast to its activation of the larval trichomes, Ubx represses leg trichomes perhaps via miR-92a (Arif et al., 2013; Crocker et al., 2015; Stern, 1998).

The size of the NV varies within and between species and these differences are associated with changes in the expression of miR-92a (Arif et al., 2013) and Ubx (Stern, 1998), respectively. 
Ubx is expressed in $D$. melanogaster T2 legs in the region of the NV, but not in the T2 legs of $D$. virilis, which has no NV (Stern, 1998). Moreover, it has been shown that Ubx contributes to differences in NV size between $D$. melanogaster and D. simulans (Stern, 1998). It was postulated that the evolution of Ubx expression in T2 legs is attributable to the presence of a T2 leg-specific enhancer of Ubx (Davis et al., 2007). However, no cis-regulatory sequences that could drive expression of $U b x$ in T2 were identified.

Here we surveyed regions of the Ubx locus where the chromatin is accessible during the window when leg trichomes are specified. We identified a novel enhancer of Ubx that can regulate trichome patterning proximally on the T2 and T3 femurs. We also identified several candidate TFs that may bind to this enhancer including Distal-less (DII), which thereby appears to regulate the trichome pattern. Our results provide a platform for better understanding the role and regulation of $U b x$ during post-embryonic development and in sculpting fine-scale adult morphology.

\section{Methods}

Fly stocks and genetics

All stocks used were kept on standard yeast extract-sucrose medium at $25^{\circ} \mathrm{C}$. Reporter lines VT42732, VT42733 and VT42734 were obtained from the Vienna Drosophila Resource Centre (VDRC). Reporter lines GMR31F12, GMR32BO3 and GMR31E11 were obtained from the FlyLight enhancer collection (Jenett et al., 2012). To test the activity of all enhancer lines, they were crossed to a UAS-stingerGFP (Bloomington stock \#65402) and/or UASshavenoid(sha) $\triangle$ UTR (Bloomington stock \#32096). To test the interaction between Ubx and miR92a, we crossed UAS-lines (Bloomington stock \#911) (Arif et al., 2013) to a pan-epidermal GAL4 driver (VT057077; VDRC) in a miR-92 loss-of-function background (Yuva-Aydemir et al., 2015). To test putative transcription factors that bind to VT42733, UAS-RNAi lines for DIl (Bloomington stock \#29337) C15 (Bloomington stock \#35018) and Bar-H2 (B-H2) (Bloomington stock \#33647) were crossed to a heat-shock GAL4 driver. White prepupae were aged for 24 hours then incubated at $37^{\circ} \mathrm{C}$ for $1 \mathrm{hr}$ to induce the heat-shock.

\section{Cloning}

Fragments UBXP1, Ubx-1, Ubx-2 and Ubx-3 were cloned from genomic DNA (D. melanogaster, Oregon R) and initially inserted into the TOPO/D vector. Primer sequences can be found in Table S1. LR gateway cloning was then used to subclone the fragments into the pBPGUw plasmid upstream of GAL4 (a gift from Gerald Rubin, Addgene plasmid \#17575). The resulting constructs were used for phiC31 mediated germline transformation into landing site 86Fb (Bloomington \#24749) by either BestGene Inc or the Cambridge injection facility.

\section{GFP and NV analysis}

White prepupae from reporter lines crossed to UAS-stingerGFP were collected and aged to between 20 and $28 \mathrm{hAPF}$, the window when T2 trichome patterning is regulated by Ubx (Stern, 1998). GFP expressing pupae were imaged on a Zeiss Axiozoom stereoscope. For the analysis of trichome patterns, T2 and T3 legs were dissected from adults and mounted in Hoyer's medium/lactic acid (1:1) and imaged under a Zeiss Axioplan microscope with a ProgRes MF cool camera (Jenaoptik). The size of the NV was measured using Fiji software (Schindelin et al., 2012) and statistical analysis was performed in R-Studio version 1.2.1335 (RCoreTeam, 2017).

\section{Identification of candidate TFs}

To identify potential TFs that bind to the Ubx NV enhancer, the JASPAR TF database was utilised (Fornes et al., 2020) with a relative profile threshold of $85 \%$ similarity. The resulting factors were compared to the RNA-seq data for T2 legs (GEO accession number GSE113240) (Kittelmann et al., 2018), and genes encoding TFs with an expression level of over 1 fragment per kb per million 
(FKPM) were scored as expressed. To further filter TFs, only those with predicted binding sites in regions of accessible chromatin, from T2 leg ATAC-seq profiling data (Table S2) (GEO accession number GSE113240) (Kittelmann et al., 2018) were selected. Further filtering of the TFs was carried out by using the UCSC genome browser (Kent et al., 2002) and regions that were identified as conserved elements (among 27 different insect species) by phastCons (Siepel et al., 2005) (Fig. S1) were used to further refine the search for candidate TFs. For each of the resulting factors, the known biological processes were extracted using FlyBase (FB2020_02) batch download (Thurmond et al., 2019). D. melanogaster genome release 6 was used in all analyses.

\section{Results}

Ubx repression of T2 leg trichomes requires miR-92a

It was previously found that $U b x$ represses the formation of trichomes on T2 femurs in a dose sensitive manner from proximal to distal (Stern, 1998). We corroborated this finding by over expressing Ubx in T2 legs using the GAL4 system, which resulted in loss of all proximal and most distal trichomes on T2 femurs (Fig. 1). As we showed previously, over-expression of miR-92a also represses T2 trichomes and, reciprocally, loss of this microRNA results in a very small NV (Arif et al., 2013; Kittelmann et al., 2018) (Fig. 1). This suggests that Ubx acts upstream of mir$92 a$ in to inhibit trichome formation. In order to test this, we over expressed Ubx in flies homozygous for a loss of function of mir-92a (and its paralogue miR-92b) (Yuva-Aydemir et al., 2015). We found that $U b x$ is unable to repress trichomes in the absence of mir-92a (Fig. 1). This suggests that $U b x$ represses trichomes by directly or indirectly activating miR-92a expression, which in turn inhibits the expression of Svb target genes including shavenoid (sha) (Arif et al., 2013; Kittelmann et al., 2018; Schertel et al., 2012). To better understand how Ubx is integrated into leg trichome GRN, we next attempted to identify cis-regulatory elements that regulate expression of this Hox gene in T2 legs.
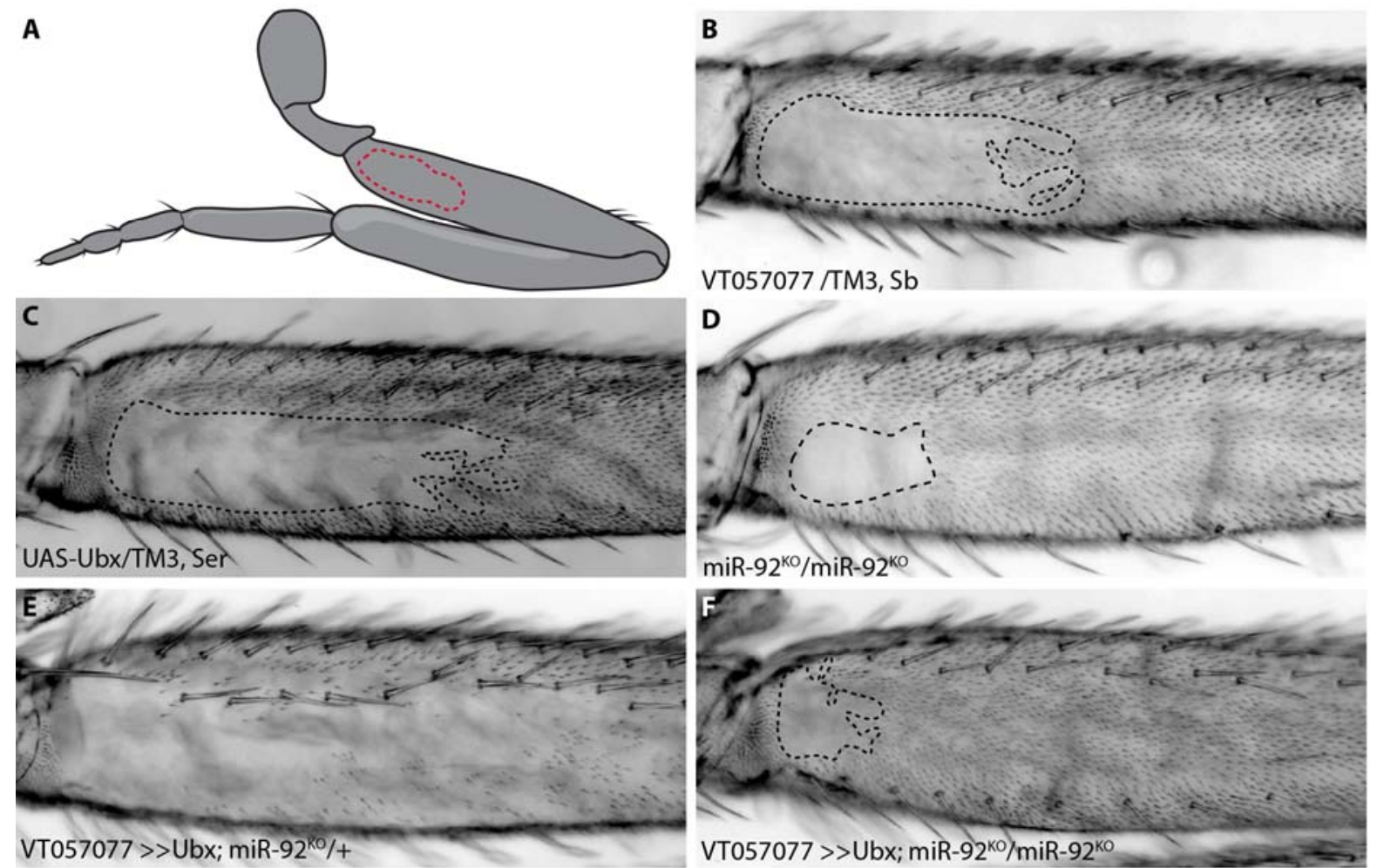

VT057077 > $>$ Ubx; miR-92 $\mathrm{ko}+$

VT057077 > $>$ Ubx; miR-92 $2^{\mathrm{KO}} / \mathrm{miR}-92^{\mathrm{KO}}$

Figure 1. Ubx requires miR-92a to repress trichomes. The naked valley is a region of trichome-free cuticle on the
posterior side of the proximal femur (outlined by dotted lines except in E) (A). The pan-epidermal GAL4 line VT057077 (B) and the UAS-Ubx line (C) both have large naked valleys, while naked cuticle is almost absent in miR-92 loss of 
function T2 legs (D). Over-expression of Ubx inhibits trichome formation on most of the femur (E), but this inhibition is restricted to the most proximal region in the $m i R-92^{\mathrm{KO}}(\mathrm{F})$.

Several regions with open chromatin drive expression in Drosophila pupal legs

To try to identify the previously predicted T2 leg Ubx enhancer, Davis et al. (2007) assayed available regulatory mutations of the Ubx locus as well as new deficiencies. This allowed them to rule out around $100 \mathrm{~kb}$ in and around the Ubx locus as containing the T2 specific enhancer. They then assayed a further $30 \mathrm{~kb}$ using reporter constructs. In total they investigated over $95 \%$ of the Ubx locus, but were unable to identify a region with T2 activity.

To follow up the work of Davis et al. (2007), we used ATAC-seq data we generated previously to generate a profile of accessible chromatin in leg cells in the developmental window when the trichome pattern is determined (Kittelmann et al., 2018) (Fig. 2). We found that the Ubx locus contains several regions of accessible chromatin, which represent known enhancers or promoters as well as putative new cis-regulatory elements. Upstream of the Ubx coding sequence we observed a series of peaks in the region of the pbx enhancer (Casanova et al., 1985; Irvine et al., 1991; Kerridge and Morata, 1982; Little et al., 1990; Morata and Kerridge, 1981). There is also a peak at the transcription start site, which presumably corresponds to the Ubx promoter (Fig. 2). Further downstream, a series of peaks that span the first and second introns is then observed. In the third and final intron there is a high peak, UBXP1, which may encompass a variably occupied CTCF site as described by Magbanua et al. (2015) and the abx polycomb response element (PRE) (Delker et al., 2019). The third intron contains another cluster of peaks which overlap with the position of $a b x$ (Simon et al., 1990) followed by further peaks in the 3' half of this intron (Fig. 2).

We then took advantage of existing reporter lines (Jenett et al., 2012; Pfeiffer et al., 2008), to assay regions containing open chromatin in the introns of Ubx for enhancer activity and specifically for expression in developing T2 legs (Fig. 2). Note that ATAC-seq peaks that correspond to known promoters and enhancers or were previously covered using reporter constructs (approximately. 3R: 16706300..16737278) by Davis et al. (2007) were not tested further in our study. In total, we tested seven regions and while three did not appear to have enhancer activity in pupae at $24 \mathrm{hAPF}$, four of the regions tested were able to drive reporter gene expression, although in all cases the expression appeared to be quite promiscuous and not limited to the developing legs (Fig. 2) 
bioRxiv preprint doi: https://doi.org/10.1101/2020.06.17.152918; this version posted July 24, 2021. The copyright holder for this preprint (which was not certified by peer review) is the author/funder, who has granted bioRxiv a license to display the preprint in perpetuity. It is made available under aCC-BY-NC-ND 4.0 International license.
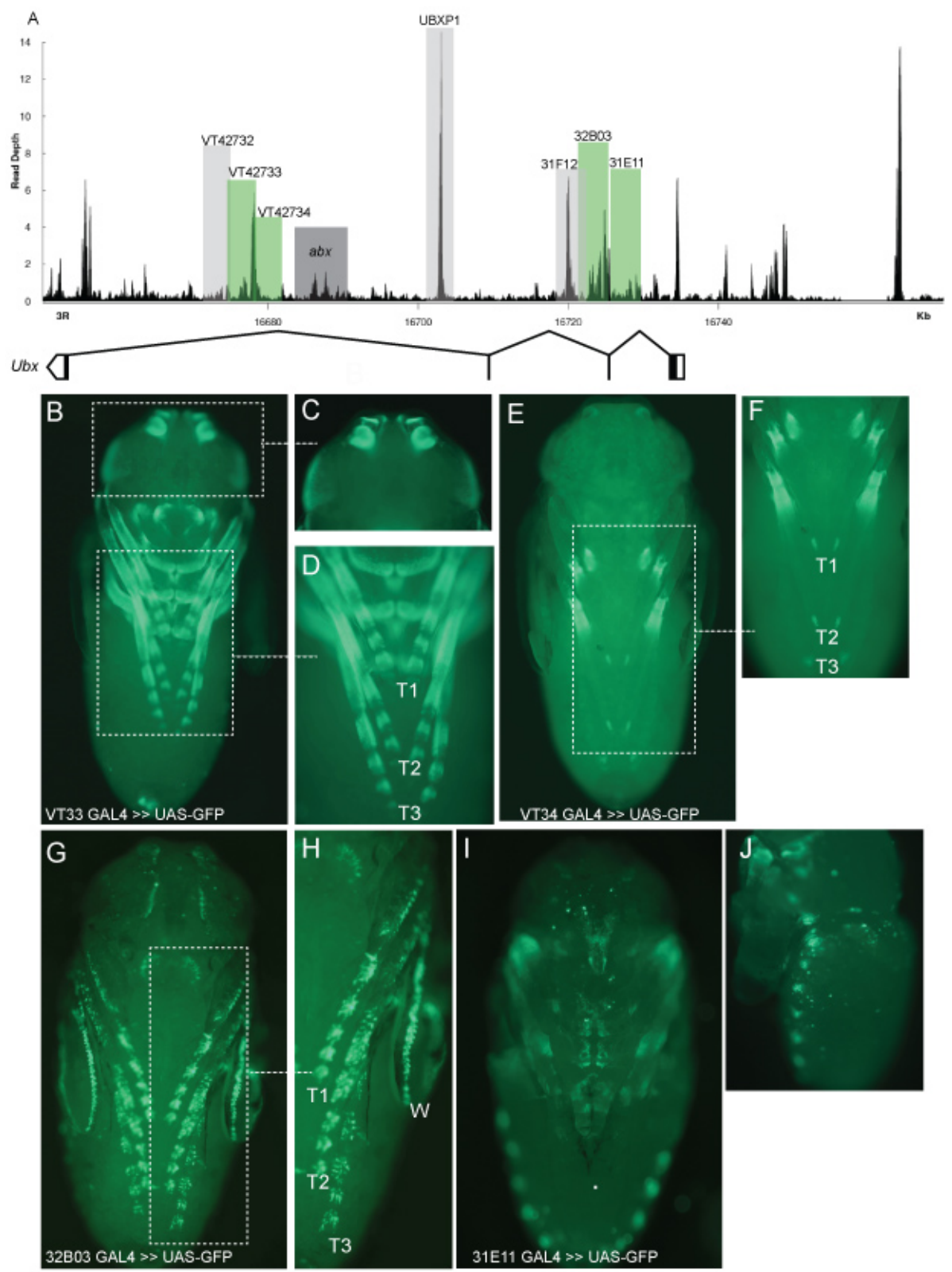

Figure 2. Testing regions of accessible chromatin at the Ubx locus for enhancer activity. (A) ATAC-seq profile for Ubx showing the location of tested regions with respect to the gene model of $U b x$. Bars above peaks indicate regions that were tested for expression in $24 \mathrm{~h}$ pupal legs. Colouring indicates activity: light grey: no expression in pupal legs at 24 hAPF, green: expression in T2 pupal legs. abx (dark grey) is a previously identified enhancer of Ubx and was therefore not tested in this study. All lines, with the exception of UBXP1, are from either the VDRC GAL4 collection or the FlyLight collection. (B-J) GFP expression in whole pupae at 24 hAPF. (B-D) VT42733 drives expression throughout the pupal legs and in the developing antenna, mouthparts, eyes, and genitals. (E-F) VT42734 drives expression predominantly in the developing legs. (G-H) GMR32B03 drives expression in the pupal limbs. This line also drives expression at the periphery of the developing wing. (I-J) GMR31E11 drives expression in the pupal legs but this is restricted to proximal limb segments. Expression is also seen on the periphery of the abdomen in distinct spots. VT33 VT42733-GAL4, VT34 - VT42734-GAL4, T1 - first leg, T2 - second leg, T3 - third leg, w - wing. 

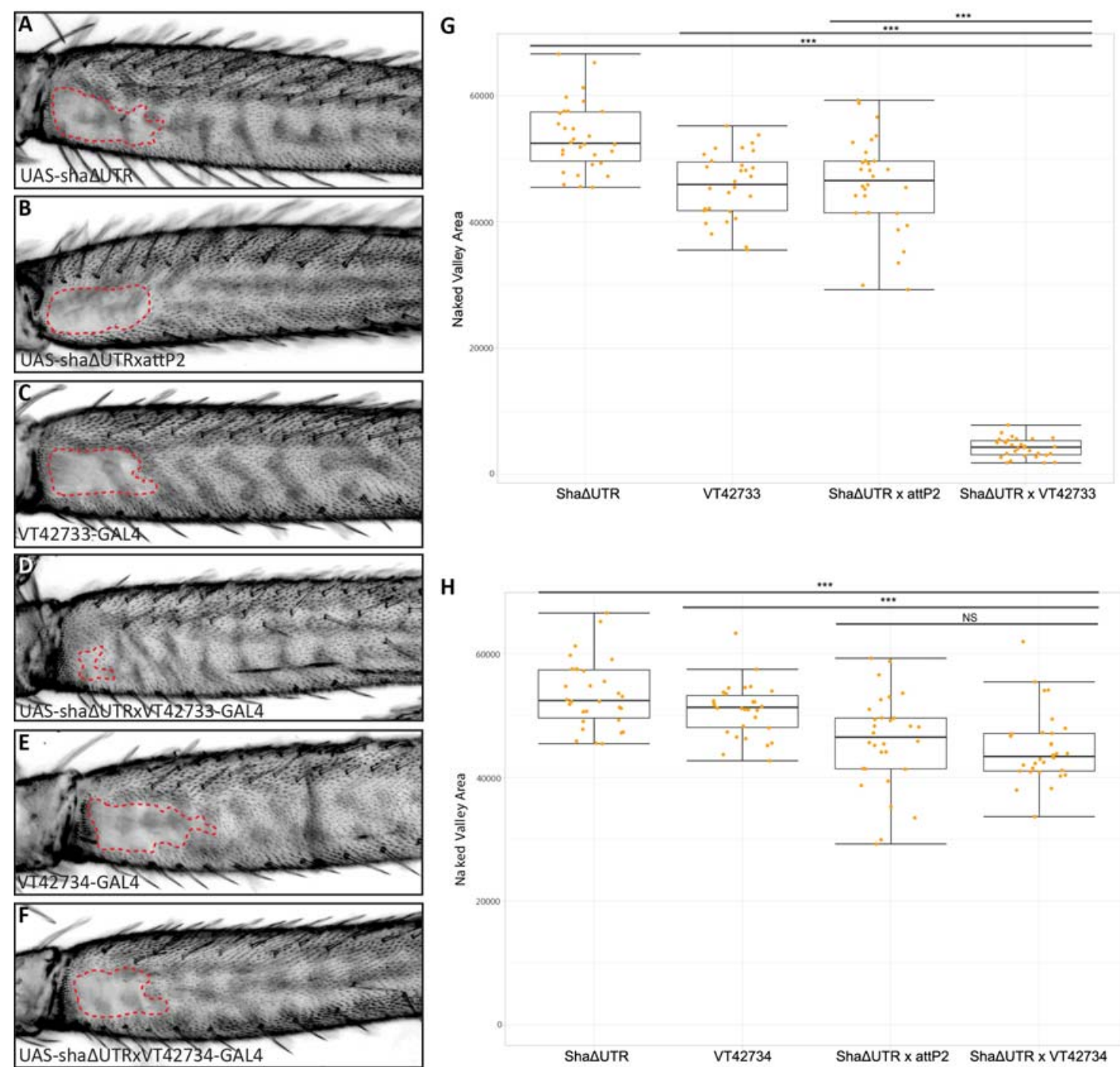

UAS-shaSUTRXVT42734-GAL4

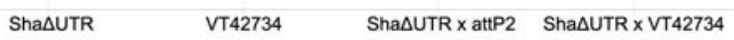

Figure 3. Functional analysis of putative enhancers. (A, B) T2 posterior femurs for the control lines used in each cross: (A) The parental line UAS-sha $\triangle$ UTR and (B) progeny from the cross between UAS-sha $\Delta U T R$ and a line with the attP landing site used to make the GAL4 insertion lines. Red dotted lines show the measured NV area. (C) Femur from the parental line VT42733 and (D) the progeny of VT42733 x UAS-shaAUTR. (E) Representative leg of the parental line VT42734 and (F) VT42734 x UAS-shaAUTR. (G) Box plot showing NV area from 30 individuals for each genotype. VT42733 induces a significant decrease in the size of the NV in comparison to all controls $\left.{ }^{* * *} p<0.001\right)$. (H) Box plot showing NV area from 30 individuals for each genotype. The size of the NV is significantly smaller for VT42734 $x$ UASsha $\triangle$ UTR when compared to both parental lines $\left({ }^{* * *} p<0.001\right)$. However, there is no significant difference between the control cross, UAS-shaAUTR $x$ attP2 and VT42734 $x$ UAS-shaAUTR (NS $p=0.887$ ). Differences in NV area were tested using Shapiro-Wilk's followed by an ANOVA. Differences between groups were then tested using Tukey's multiple comparison test.

Ubx VT42733 is capable of inducing trichome formation in the NV

We next tested if the four regions that drive GFP expression in pupal legs also have the ability to functionally specify the trichome pattern seen on second legs. To do this we crossed the driver lines to UAS-sha $\triangle U T R$, which overrides trichome repression by miR-92a and leads to the formation of trichomes on normally naked cuticle (Arif et al., 2013). Therefore, in this assay, enhancer regions that are active in the NV at the correct time will generate trichomes where there is normally naked cuticle (Fig. 3). GMR32B03 and GMR31E11 drive GFP expression in developing pupal legs (Fig. 2) but they did not have any effect on trichome pattern in the NV. However, VT42733 was able to induce the formation of trichomes in the NV, resulting in a dramatic decrease in the size of the patch of naked cuticle (Fig. 3). VT42734, which overlaps with 
VT42733 by approximately 400 bp (Fig. 2), also produced a significantly reduced size of the NV in comparison to both parental controls and therefore may drive weak expression in this region. However, the NV of VT42734xUAS-sha $\triangle U T R x$ attP progeny were not significantly different to those of the control cross, attpxUAS-sha $\triangle U T R$ (Fig. 3). These results suggest that region VT42733 regulates $U b x$ expression in the posterior femur and encompasses a novel T2 leg enhancer for this Hox gene. Importantly, we noticed that while VT42733 greatly reduces the size of the NV in this assay, a small patch of naked cuticle remains on the ventral of the T2 posterior femur (Fig. 3), which is consistent with the known activity of Ubx in the T2 leg (Davis et al., 2007). Furthermore, VT42733 drives expression in the proximal regions of the anterior and posterior compartments of T3 legs, again reflecting regions of Ubx activity in these legs, albeit not extending as distally as the effect of this gene on the posterior T3 femur (Fig. S2) (Davis et al., 2007). Taken together these results evidence that the Ubx leg enhancer we have identified captures endogenous activity of this Hox gene in the NV region of T2 legs as well as proximally on T3 legs.

\section{Identifying a minimal Ubx T2 leg enhancer}

To further delineate the Ubx NV enhancer region, VT42733 was broken down into three overlapping fragments of around $700 \mathrm{bp}$ : Ubx-1, Ubx-2, and Ubx-3 (Fig. 4). All three lines were able to drive GFP expression in developing pupae: Ubx-1 drives expression in legs, antennae and developing eyes (Fig. 4), Ubx-2 drives a more restricted expression pattern limited to small patch in the pupal legs and in the head (Fig. 4), and Ubx-3 also drives expression in the legs, but predominantly in the head and thorax. Interestingly, Ubx-3 also drives a stripe-like pattern on the ventral side of the abdomen, which was not seen in any of the other driver lines tested (Fig. 4).

To further test the functionality of these new smaller enhancer regions, they were crossed to UAS-sha $\triangle$ UTR. We observed that Ubx-1 is able to drive trichomes in the NV albeit in a patchy and irregular pattern (Fig. 4). However, none of the other lines had any detectable activity in this assay (Fig. 4). This suggests the main enhancer activity of this region in the developing T2 legs is determined by TF binding sites (TFBS) in Ubx-1. We also observed that Ubx-1 is able to recapitulate some of the activity of $U b x$ in T3 trichome patterning as described in Davis et al. (2007) (Fig. S2). 

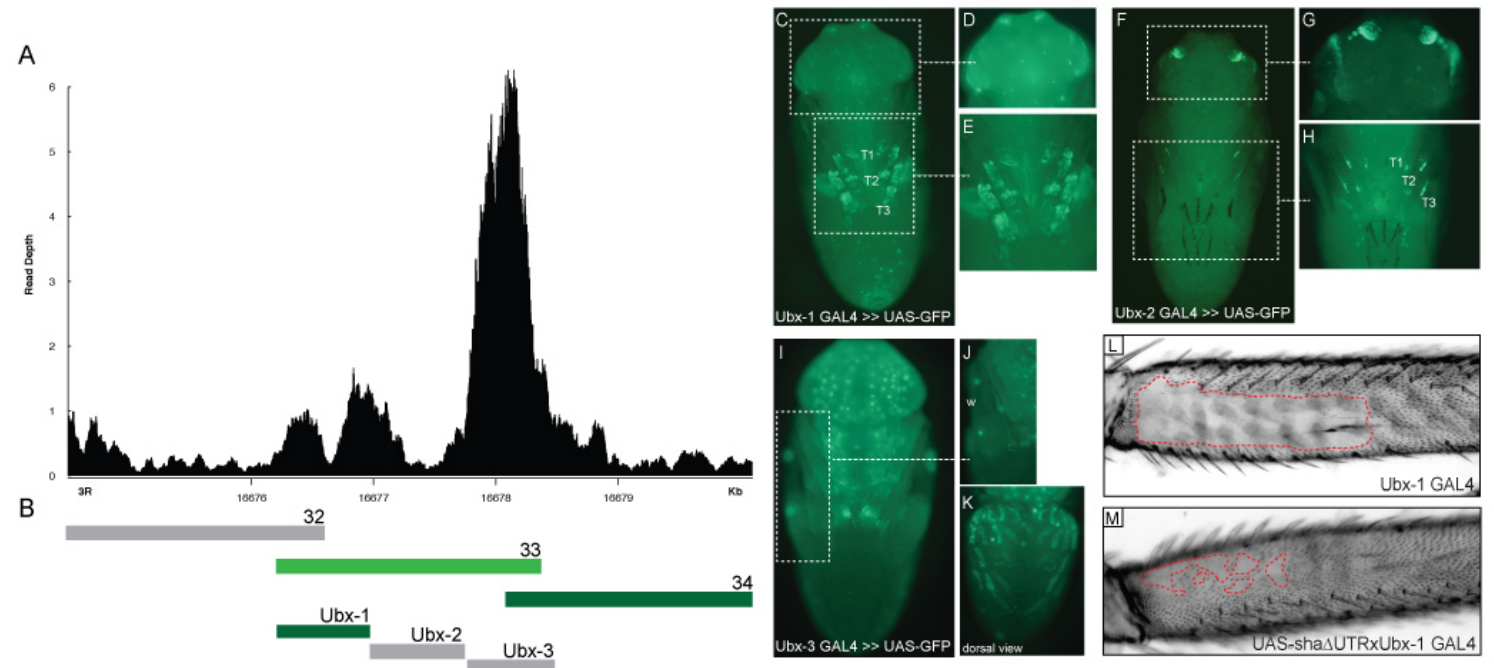

Figure 4. Characterisation of fragments of VT42733. (A) The ATAC-seq profile corresponding to VT42733 shows several distinct peaks of open chromatin. (B) Location of the GAL4 lines in relation to each other. VT42732, VT42733 and VT42734 each overlap by 400 bp. Ubx-1, Ubx-2 and Ubx-3 each overlap by $\sim 10$ bp. Grey bars represent lines that did not have a detectable effect on trichome formation in the NV when combined with UAS-sha $\Delta$ UTR, dark green bars indicate lines which had a minor effect in the NV and the light green bar represents VT42733 which drives expression throughout most of the NV. (C-K) Expression of driver lines Ubx-1, Ubx-2 and Ubx-3 in pupae at 24 hAPF. Expression of Ubx-1 seems restricted to the legs of pupae (C-E). Ubx-2 also expresses in pupal legs but is more restricted than $\mathrm{Ubx}-1(\mathrm{~F}, \mathrm{H})$ and also drives strong expression in the developing antenna ( $\mathrm{F}-\mathrm{G})$. Ubx-3 expression is much more restricted to the head tissue (I) with some dots of expression in the developing wing ( $\mathrm{J}$ ) and a stripe like pattern on the dorsal abdomen $(\mathrm{K})$. Only Ubx-1 is capable of initiating trichome development in the NV $(\mathrm{L}, \mathrm{M})$. Parental control lines (L), Fig. 3A.

\section{Analysis of transcription factor binding sites in the NV enhancer}

To further characterise the Ubx NV enhancer, we carried out motif analysis to identify TFs that may bind to this region. To focus on binding sites for TFs that are expressed at the time of trichome development, we cross-referenced previously generated RNA-seq data for T2 legs (Kittelmann et al., 2018) with the JASPAR database (Fornes et al., 2020) (with the caveat that the JASPAR database does not contain an exhaustive list of all Drosophila TFs). Using a threshold of 85\% similarity and focussing only on TFs expressed above a 1 FPKM threshold in T2 legs, 64 TFs were found to have predicted binding sites in the VT42733 region. We then applied two more approaches to further filter these candidates. First, we used our ATAC-seq data to remove TFs with predicted binding sites located only in the inaccessible chromatin of region VT42733. Second, we used phastCons from the UCSC genome browser (Siepel et al., 2005) to identify conserved sequences within VT42733 (Fig. S1). Using this method, we identified a total of 52 factors (33 with predicted binding sites in Ubx-1) that are expressed in T2 legs and predicted to bind to conserved, accessible regions in the VT42733 enhancer (Fig. 5; Supplementary File 1). We then used FlyBase to survey the 52 TFs for known roles in leg development leaving a shortlist of 14 (eight of which also have predicted binding sites in Ubx-1) including DII, Extradenticle, C15, BarH1 (B-H1) and B-H2 (Fig. 5; Table S1). This list also include several binding sites for Ubx itself suggesting there may be Ubx autoregulation in legs as found in other tissues (Fig. 5; Table S1) (Delker et al., 2019). 
bioRxiv preprint doi: https://doi.org/10.1101/2020.06.17.152918; this version posted July 24, 2021. The copyright holder for this preprint (which was not certified by peer review) is the author/funder, who has granted bioRxiv a license to display the preprint in perpetuity. It is made
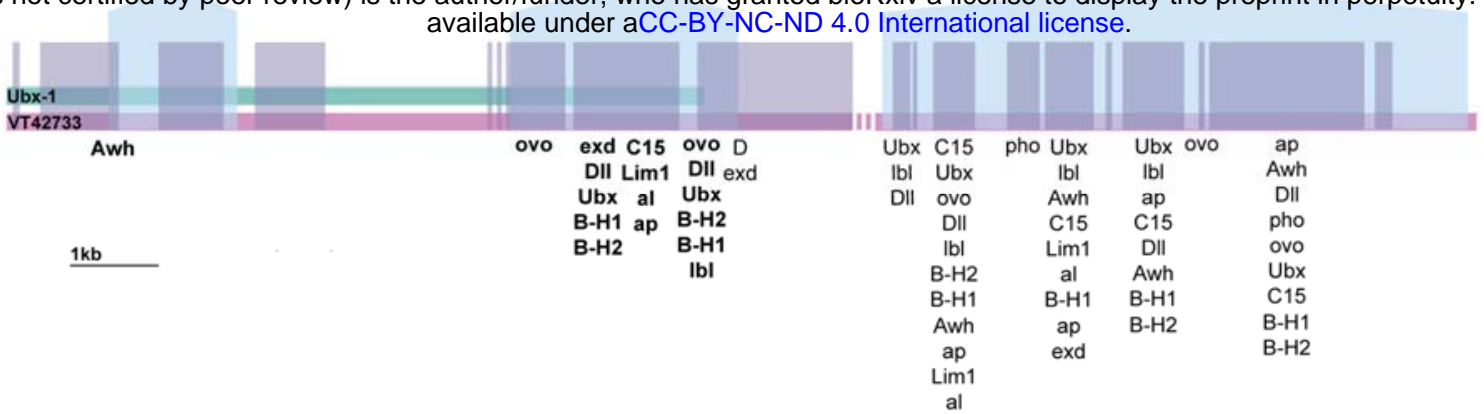

Figure 5. Analysis of potential TFBS in the NV enhancer region. TFBS as predicted by JASPAR and their positions in both VT42733 (pink bar) and the smaller fragment Ubx-1 (green bar) both of which are active in the NV. The light blue arrows represent areas of open chromatin according to the ATAC-seq data (Kittelmann et al., 2018). The light purple boxes represent conserved sequences according to UCSC phastCons genome track. Note that a portion of VT42733 is excluded from the figure because no open chromatin is found there (represented by pink dots: 3R: 16677172..16677767). Predicted TFBS as determined by JASPAR that are found in both areas of conservation and open chromatin and have a characterised role in leg development are shown underneath their respective locations. TFBS in bold text are those located in Ubx-1. See also Supplementary File 1.

\section{Testing the function of candidate TFs during leg development}

We next tested the role of $\mathrm{DIl}, \mathrm{C} 15$ and $\mathrm{B}-\mathrm{H} 2$ in leg trichome patterning by knocking-down their expression in the window of time when trichomes develop (24 hAPF) using the GAL4 system to apply heat shock induced RNAi. Knockdown of $C 15$ and $B-H 2$ had no effect on trichome pattern (not shown). However, upon knockdown of DIl we observed a striking loss of trichomes on the T2 femur, although the effect was somewhat variable probably due to the efficiency of RNAi induction (Fig. 6). These data, together with the motif predictions, suggest that DII is involved in trichome formation perhaps by directly regulating Ubx via the NV enhancer. Moreover, this would suggest that DII represses Ubx in this context, since Ubx inhibits trichome development and fewer trichomes develop on the T2 femur when DII is knocked-down (Fig. 6).
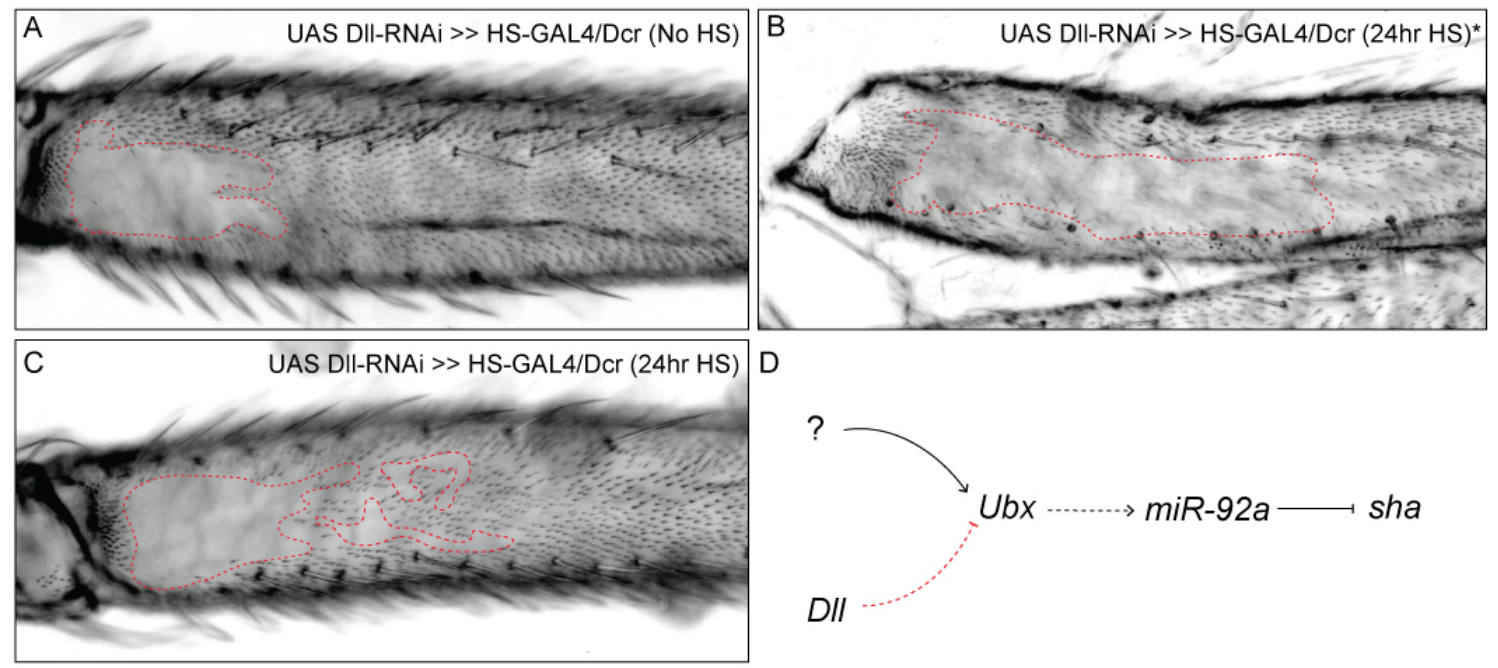

Figure 6. RNAi knock-down of DII reveals potential role in the leg GRN. (A) Control line UAS-DII-RNAi combined with Heat-shock (HS)-GAL4/Dicer. This line was not heat-shocked and displays a medium sized NV. (B-C) When the heat-shock is performed at 24 hAPF two classes of phenotype were observed. Some adults were unable to eclose and when dissected from their pupal cases, their legs display a dramatic increase in the size of the NV (B). The legs of those that eclosed displayed a patchy and irregular pattern of trichomes in the NV region and extending distally. (D) Schematic of the gene regulatory interactions tested in this study. Expression of Ubx could be directly repressed by DIl, while Ubx activates directly or indirectly miR-92a, which represses sha, a gene crucial for trichome development.

\section{Discussion}

We searched for an Ubx T2 leg enhancer guided by regions of accessible chromatin in cells in this tissue, identified using ATAC-seq. Four of the seven lines from existing reporter construct 
collections tested were able to drive detectable GFP expression in the developing pupal legs, but none of the lines drove expression specific to just the NV. However, using functional testing, we found that the $2.2 \mathrm{~kb}$ region VT42733 has enhancer activity in NV cells during the correct developmental time point (Fig. 3). The adjacent region, VT42734, may also have weak activity in the proximal part of the T2 femur (Fig. 3). Regions VT42733 and VT42734 overlap by $400 \mathrm{bp}$ and so it is possible that VT42734 is able to carry out part of the function of VT42733 due to this overlap (Fig. 4). Analysis of sub-fragments of VT42733 showed that a 700 bp region, Ubx-1, is also active in NV cells, but this activity is weaker than the full VT42733 sequence. Therefore, Ubx-1 likely contains only a subset of the binding sites responsible for the stronger activity of VT42733. For example, Ubx-1 contains fewer predicted DII and Exd binding sites in comparison to the full VT42733 region (Table S1). However, any additional binding sites in regions of VT42733 outwith Ubx-1 appear insufficient to drive expression on their own, since Ubx-2 and Ubx-3 had no detectable functional activity. Taken together, these results indicate that the Ubx T2 enhancer is located in region VT42733 with some binding sites concentrated in region Ubx- 1. Importantly, while VT42733 and Ubx-1 are able to drive expression in the proximal femur, they are inactive in the ventral part of the posterior T2 femur (Fig. 2). This was particularly evident for VT42733 when combined with UAS-sha $\triangle$ UTR, which resulted in the entire posterior femur being covered in trichomes apart from a small ventral region (Fig. 2). This is consistent with previous studies that showed while Ubx represses trichomes on the posterior T2 femurs, it is inactive in these ventral cells, and even in the absence of $U b x$, this region of the cuticle fails to differentiate trichomes (Davis et al., 2007; Stern, 2003). Indeed, this region also stays trichome-free in a miR92 loss of function line (Fig. 1D), indicating that repression of trichomes in these cells is independent of $U b x$ and miR-92a. The activity of VT42733 is also consistent with Ubx function on the proximal region of T3 (Davis et al., 2007). This suggests that the enhancer we have identified does indeed recapitulate the expression of $U b x$ in T2 and to some extent T3. Interestingly, FAIRE-seq to assay the open chromatin in developing halteres and wings, revealed the while the $a b x$ region is accessible there was no distinctive peak in the region of the NV enhancer we have discovered aided by T2 leg-specific ATAC-seq (Delker et al., 2019; McKay and Lieb, 2013). This suggests that while the enhancer we have identified is accessible and active in legs it is not used in the developing halteres or wings.

Davis et al. (2007) previously surveyed most of the third intron of Ubx, including the VT42733 region, for a T2 leg enhancer. However, they did not identify any regions with pupal leg activity although they found that $a b x$ (Fig. 2) is required for earlier expression during T2 development consistent with previous studies (Casanova et al., 1985; Davis et al., 2007; Kerridge and Morata, 1982; Peifer and Bender, 1986). This apparent inconsistency with our results could be explained by the different methods used to locate the enhancer. While we used reporter constructs encompassing regions of accessible chromatin in T2 pupal legs to discover that VT42733 is able to drive expression in NV cells, Davis et al. (2007) covered this region using deficiencies and found no effect on the trichome patterning of the T2 femur. This suggests that VT42733 is able to drive expression in NV cells but removal of this region does not affect the trichome pattern perhaps because of compensation by additional binding sites located elsewhere in the Ubx locus. To more directly test this, it would be interesting to precisely delete the NV enhancer from the endogenous location instead of using large deficiencies of the Ubx locus that likely have pleiotropic effects and perhaps even result in prepupal lethality.

Recent analysis of the $a b x$ enhancer resulted in similar findings to our study and those of Davis et al. (2007). Delker et al. (2019) showed that a reporter construct with a minimal region of $531 \mathrm{bp}$ of the $a b x$ enhancer that contains autoregulatory Ubx-Exd binding sites is able to recapitulate differential Ubx expression in proximal versus distal cells of the developing halteres (Delker et al., 2019). However, deletion of this region using CRISPR/Cas9 had no effect on this expression pattern. The authors concluded that there are likely additional binding sites elsewhere 
and potentially even scattered throughout the Ubx locus that contribute to its differential expression in the halteres (Delker et al., 2019).

It is clear that many of the fragments of the Ubx locus that we tested for enhancer activity, including the NV enhancer VT42733, are active in other pupal tissues that express Ubx such as the T3 legs but also in places that are not known to normally express Ubx, for example the T1 legs. This suggests that these fragments exclude binding sites for TFs that repress Ubx in these tissues or other cis-regulatory elements like boundary elements that restrict Ubx expression to the correct locations.

Ectopic expression has been observed previously with reporter constructs for Hox genes such as Scr and Ubx. abx fragments drive ectopic expression in imaginal discs that do not normally express $U b x$ and this has been suggested to be a consequence of their exclusion of a nearby PRE (Delker et al., 2019), and potentially the variably occupied CTCF site in the third intron (Fig. 2) (Magbanua et al., 2015). Furthermore, reporter constructs for recently identified Scr enhancers that reproduce expression of this Hox gene in T1 also appear to be ectopically active in other legs where Scr is normally repressed (Eksi et al., 2018). It has also been suggested that these reporters contain binding sites that facilitate expression in all legs but they are missing silencer elements that normally restrict Scr to T1 (Eksi et al., 2018), which could also be the case with the Ubx leg enhancer.

Our identification of a T2 enhancer of Ubx allowed us to begin to decipher how this Hox gene is regulated in a specific developmental context and to further explore the topology of the surrounding GRN. Analysis of TFBS in conserved sequences in open chromatin compared with the activity of VT42733 and its derivatives Ubx-1, Ubx-2 and Ubx-3 suggests this enhancer is regulated by TFs including Exd and DII (Fig. 5). Intriguingly, Exd is a well-known cofactor of Ubx in many processes including the development of leg imaginal discs (Mann, 1995), but we have not yet tested its role in leg trichome patterning.

Our data also suggest that DII may directly regulate the Ubx T2 enhancer via up to eight predicted binding sites. Furthermore, it appears that DII represses Ubx since knockdown of DII removes trichomes, while trichomes are gained when Ubx activity is reduced (Stern, 1998). Interestingly, there is evidence that DII represses other genes during leg development including serrate (Rauskolb, 2001). We suggest that DIl-mediated repression of Ubx may help to ensure the generation of trichomes on the distal region of the femur while Ubx activates miR-92a more proximally to repress trichomes and generate the NV. However, a more detailed understanding of these regulatory interactions requires assaying whether DII and Ubx bind directly to the Ubx and mir-92a enhancers, respectively. Intriguingly, our binding site predictions also suggest that there might be autoregulation of Ubx through the NV enhancer as has been described, for example, for fine tuning expression of this gene in developing halteres (Delker et al., 2019), but this needs to be tested further. The identification and validation of other TFs that bind to the NV enhancer to positively regulate $U b x$ is also required to fully understand its function in the specification of trichome patterning on T2 legs.

In conclusion, we have identified a T2 leg enhancer of Ubx that drives expression to sculpt the fine-scale morphology of this appendage. This provides new insights into the regulation of this Hox gene during postembryonic development and will serve as a platform to better understand how it is wired into the wider leg trichome GRN.

\section{Acknowledgements}

ADB was funded by a BBSRC DTP studentship and SK by a DFG Research Fellowships (Ki 1831/1-1). Stocks obtained from the Bloomington Drosophila Stock Center (NIH P40OD018537) were used in this study. We thank Marianne Yoth for assistance with experiments. 


\section{References}

Akam, M., 1998a. Hox genes, homeosis and the evolution of segment identity: no need for hopeless monsters. International Journal of Developmental Biology 42, 445-451, SI 1998.

Akam, M., 1998b. Hox genes: From master genes to micromanagers. Current Biology 8, R676-678.

Akam, M.E., Martinez-Arias, A., 1985. The distribution of Ultrabithorax transcripts in Drosophila embryos. The EMBO journal 4, 1689-1700.

Arif, S., Kittelmann, S., McGregor, A.P., 2015. From shavenbaby to the naked valley: trichome formation as a model for evolutionary developmental biology. Evolution \& development 17, 120-126.

Arif, S., Murat, S., Almudi, I., Nunes, M.D., Bortolamiol-Becet, D., McGregor, N.S., Currie, J.M., Hughes, H., Ronshaugen, M., Sucena, E., Lai, E.C., Schlotterer, C., McGregor, A.P., 2013. Evolution of mir-92a Underlies Natural Morphological Variation in Drosophila melanogaster. Current biology : CB 23, 523-528.

Balmert, A., Florian Bohn, H., Ditsche-Kuru, P., Barthlott, W., 2011. Dry under water: comparative morphology and functional aspects of air-retaining insect surfaces. Journal of morphology $272,442-451$.

Bender, W., Akam, M., Karch, F., Beachy, P.A., Peifer, M., Spierer, P., Lewis, E.B., Hogness, D.S., 1983. Molecular Genetics of the Bithorax Complex in Drosophila melanogaster. Science (New York, N.Y.) 221, 23-29.

Brodu, V., Elstob, P.R., Gould, A.P., 2002. abdominal A specifies one cell type in Drosophila by regulating one principal target gene. Development (Cambridge, England) 129, 2957-2963.

Buffry, A.D., Mendes, C.C., McGregor, A.P., 2016. The Functionality and Evolution of Eukaryotic Transcriptional Enhancers. Adv Genet 96, 143-206.

Carroll, S.B., Grenier, J.K., Weatherbee, S.D., 2005. From DNA to Diversity, Molecular Genetics and the Evolution of Animal Design, 2nd ed. Blackwell Publishing, Malden.

Casanova, J., Sánchez-Herrero, E., Morata, G., 1985. Prothoracic transformation and functional structure of the Ultrabithorax gene of Drosophila. Cell 42, 663-669.

Castelli-Gair, J., Akam, M., 1995. How the Hox gene Ultrabithorax specifies two different segments: The significance of spatial and temporal regulation within metameres. Development (Cambridge, England) 121, 2973-2982.

Chanut-Delalande, H., Fernandes, I., Roch, F., Payre, F., Plaza, S., 2006. Shavenbaby couples patterning to epidermal cell shape control. PLoS biology 4, e290.

Crocker, J., Abe, N., Rinaldi, L., McGregor, A.P., Frankel, N., Wang, S., Alsawadi, A., Valenti, P., Plaza, S., Payre, F., Mann, R.S., Stern, D.L., 2015. Low affinity binding site clusters confer hox specificity and regulatory robustness. Cell 160, 191-203.

Davis, G.K., Srinivasan, D.G., Wittkopp, P.J., Stern, D.L., 2007. The function and regulation of Ultrabithorax in the legs of Drosophila melanogaster. Developmental biology 308, 621-631.

Delker, R.K., Ranade, V., Loker, R., Voutev, R., Mann, R.S., 2019. Low affinity binding sites in an activating CRM mediate negative autoregulation of the Drosophila Hox gene Ultrabithorax. PLoS genetics 15, e1008444.

Delon, I., Chanut-Delalande, H., Payre, F., 2003. The Ovo/Shavenbaby transcription factor specifies actin remodelling during epidermal differentiation in Drosophila. Mechanisms of development 120, 747-758.

Diaz-de-la-Loza, M.D., Loker, R., Mann, R.S., Thompson, B.J., 2020. Control of tissue morphogenesis by the HOX gene Ultrabithorax. Development (Cambridge, England) 147. 
Ditsche-Kuru, P., Schneider, E.S., Melskotte, J.E., Brede, M., Leder, A., Barthlott, W., 2011.

Superhydrophobic surfaces of the water bug Notonecta glauca: a model for friction reduction and air retention. Beilstein J Nanotechnol 2, 137-144.

Eksi, S.E., Barmina, O., McCallough, C.L., Kopp, A., Orenic, T.V., 2018. A Distalless-responsive enhancer of the Hox gene Sex combs reduced is required for segment- and sex-specific sensory organ development in Drosophila. PLoS genetics 14, e1007320.

Estacio-Gómez, A., Moris-Sanz, M., Schäfer, A.K., Perea, D., Herrero, P., Díaz-Benjumea, F.J., 2013. Bithorax-complex genes sculpt the pattern of leucokinergic neurons in the Drosophila central nervous system. Development (Cambridge, England) 140, 2139-2148.

Fornes, O., Castro-Mondragon, J.A., Khan, A., van der Lee, R., Zhang, X., Richmond, P.A., Modi, B.P., Correard, S., Gheorghe, M., Baranašić, D., Santana-Garcia, W., Tan, G., Chèneby, J., Ballester, B., Parcy, F., Sandelin, A., Lenhard, B., Wasserman, W.W., Mathelier, A., 2020. JASPAR 2020: update of the openaccess database of transcription factor binding profiles. Nucleic acids research 48, D87-D92.

Frankel, N., Erezyilmaz, D.F., McGregor, A.P., Wang, S., Payre, F., Stern, D.L., 2011. Morphological evolution caused by many subtle-effect substitutions in regulatory DNA. Nature 474, 598-603.

Frankel, N., Wang, S., Stern, D.L., 2012. Conserved regulatory architecture underlies parallel genetic changes and convergent phenotypic evolution. Proceedings of the National Academy of Sciences of the United States of America 109, 20975-20979.

Halfon, M.S., 2019. Studying Transcriptional Enhancers: The Founder Fallacy, Validation Creep, and Other Biases. Trends in genetics : TIG 35, 93-103.

Hombría, J.C., Lovegrove, B., 2003. Beyond homeosis--HOX function in morphogenesis and organogenesis. Differentiation; research in biological diversity 71, 461-476.

Hueber, S.D., Lohmann, I., 2008. Shaping segments: Hox gene function in the genomic age. BioEssays : news and reviews in molecular, cellular and developmental biology 30, 965-979.

Irvine, K.D., Botas, J., Jha, S., Mann, R.S., Hogness, D.S., 1993. Negative autoregulation by Ultrabithorax controls the level and pattern of its expression. Development (Cambridge, England) 117, 387-399.

Irvine, K.D., Helfand, S.L., Hogness, D.S., 1991. The large upstream control region of the Drosophila homeotic gene Ultrabithorax. Development (Cambridge, England) 111, 407-424.

Jenett, A., Rubin, G.M., Ngo, T.T., Shepherd, D., Murphy, C., Dionne, H., Pfeiffer, B.D., Cavallaro, A., Hall, D., Jeter, J., lyer, N., Fetter, D., Hausenfluck, J.H., Peng, H., Trautman, E.T., Svirskas, R.R., Myers, E.W., Iminski, Z.R., Aso, Y., DePasquale, G.M., Enos, A., Hulamm, P., Lam, S.C., Li, H.H., Laverty, T.R., Long, F., Qu, L., Murphy, S.D., Rokicki, K., Safford, T., Shaw, K., Simpson, J.H., Sowell, A., Tae, S., Yu, Y., Zugates, C.T., 2012. A GAL4-driver line resource for Drosophila neurobiology. Cell reports 2, 991-1001.

Kannan, R., Berger, C., Myneni, S., Technau, G.M., Shashidhara, L.S., 2010. Abdominal-A mediated repression of Cyclin E expression during cell-fate specification in the Drosophila central nervous system. Mechanisms of development 127, 137-145.

Kent, W.J., Sugnet, C.W., Furey, T.S., Roskin, K.M., Pringle, T.H., Zahler, A.M., Haussler, D., 2002. The human genome browser at UCSC. Genome research 12, 996-1006.

Kerridge, S., Morata, G., 1982. Developmental effects of some newly induced Ultrabithorax alleles of Drosophila. Journal of embryology and experimental morphology 68, 211-234.

Kittelmann, S., Buffry, A.D., Franke, F.A., Almudi, I., Yoth, M., Sabaris, G., Couso, J.P., Nunes, M.D.S., Frankel, N., Gomez-Skarmeta, J.L., Pueyo-Marques, J., Arif, S., McGregor, A.P., 2018. Gene regulatory network architecture in different developmental contexts influences the genetic basis of morphological evolution. PLoS genetics 14, e1007375.

Krumlauf, R., 2018. Hox genes, clusters and collinearity. The International journal of developmental biology 62, 659-663. 
Lewis, E.B., 1978. A gene complex controlling segmentation in Drosophila. Nature 276, 565-570.

Little, J.W., Byrd, C.A., Brower, D.L., 1990. Effect of abx, bx and pbx mutations on expression of homeotic genes in Drosophila larvae. Genetics 124, 899-908.

Maeda, R.K., Karch, F., 2006. The ABC of the BX-C: the bithorax complex explained. Development (Cambridge, England) 133, 1413-1422.

Magbanua, J.P., Runneburger, E., Russell, S., White, R., 2015. A variably occupied CTCF binding site in the ultrabithorax gene in the Drosophila bithorax complex. Molecular and cellular biology 35, 318-330.

Mann, R.S., 1995. The specificity of homeotic gene function. BioEssays : news and reviews in molecular, cellular and developmental biology 17, 855-863.

McGinnis, W., Krumlauf, R., 1992. Homeobox genes and axial patterning. Cell 68, 283-302.

McGregor, A.P., Orgogozo, V., Delon, I., Zanet, J., Srinivasan, D.G., Payre, F., Stern, D.L., 2007. Morphological evolution through multiple cis-regulatory mutations at a single gene. Nature 448, 587-590.

McKay, D.J, Lieb J.D., 2013. A common set of DNA regulatory elements shapes Drosophila appendages. Dev Cell 27, 306-18.

Menoret, D., Santolini, M., Fernandes, I., Spokony, R., Zanet, J., Gonzalez, I., Latapie, Y., Ferrer, P., Rouault, H., White, K.P., Besse, P., Hakim, V., Aerts, S., Payre, F., Plaza, S., 2013. Genome-wide analyses of Shavenbaby target genes reveals distinct features of enhancer organization. Genome biology 14, R86.

Morata, G., Kerridge, S., 1981. Sequential functions of the bithorax complex of Drosophila. Nature 290, 778-781.

Müller, J., Bienz, M., 1991. Long range repression conferring boundaries of Ultrabithorax expression in the Drosophila embryo. The EMBO journal 10, 3147-3155.

Pavlopoulos, A., Akam, M., 2011. Hox gene Ultrabithorax regulates distinct sets of target genes at successive stages of Drosophila haltere morphogenesis. Proceedings of the National Academy of Sciences of the United States of America 108, 2855-2860.

Pearson, J.C., Lemons, D., McGinnis, W., 2005. Modulating Hox gene functions during animal body patterning. Nature reviews. Genetics 6, 893-904.

Peifer, M., Bender, W., 1986. The anterobithorax and bithorax mutations of the bithorax complex. The EMBO journal 5, 2293-2303.

Pfeiffer, B.D., Jenett, A., Hammonds, A.S., Ngo, T.T., Misra, S., Murphy, C., Scully, A., Carlson, J.W., Wan, K.H., Laverty, T.R., Mungall, C., Svirskas, R., Kadonaga, J.T., Doe, C.Q., Eisen, M.B., Celniker, S.E., Rubin, G.M., 2008. Tools for neuroanatomy and neurogenetics in Drosophila. Proceedings of the National Academy of Sciences of the United States of America 105, 9715-9720.

Preger-Ben Noon, E., Davis, F.P., Stern, D.L., 2016. Evolved Repression Overcomes Enhancer Robustness. Developmental cell 39, 572-584.

Rauskolb, C., 2001. The establishment of segmentation in the Drosophila leg. Development (Cambridge, England) 128, 4511-4521.

RCoreTeam, 2017. R: A language and environment for statistical computing. R Foundation for Statistical Computing.

Roch, F., Akam, M., 2000. Ultrabithorax and the control of cell morphology in Drosophila halteres. Development (Cambridge, England) 127, 97-107.

Schertel, C., Rutishauser, T., Forstemann, K., Basler, K., 2012. Functional characterization of Drosophila microRNAs by a novel in vivo library. Genetics 192, 1543-1552. 
Schindelin, J., Arganda-Carreras, I., Frise, E., Kaynig, V., Longair, M., Pietzsch, T., Preibisch, S., Rueden, C., Saalfeld, S., Schmid, B., Tinevez, J.Y., White, D.J., Hartenstein, V., Eliceiri, K., Tomancak, P., Cardona, A., 2012. Fiji: an open-source platform for biological-image analysis. Nature methods 9, 676-682.

Siepel, A., Bejerano, G., Pedersen, J.S., Hinrichs, A.S., Hou, M., Rosenbloom, K., Clawson, H., Spieth, J., Hillier, L.W., Richards, S., Weinstock, G.M., Wilson, R.K., Gibbs, R.A., Kent, W.J., Miller, W., Haussler, D., 2005. Evolutionarily conserved elements in vertebrate, insect, worm, and yeast genomes. Genome research 15, 1034-1050.

Simon, J., Peifer, M., Bender, W., O'Connor, M., 1990. Regulatory elements of the bithorax complex that control expression along the anterior-posterior axis. The EMBO journal 9, 3945-3956.

Stern, D.L., 1998. A role of Ultrabithorax in morphological differences between Drosophila species. Nature 396, 463-466.

Stern, D.L., 2003. The Hox gene Ultrabithorax modulates the shape and size of the third leg of Drosophila by influencing diverse mechanisms. Developmental biology 256, 355-366.

Stern, D.L., Frankel, N., 2013. The structure and evolution of cis-regulatory regions: the shavenbaby story. Philos Trans R Soc Lond B Biol Sci 368, 20130028.

Struhl, G., 1982. Genes controlling segmental specification in the Drosophila thorax. Proceedings of the National Academy of Sciences of the United States of America 79, 7380-7384.

Sucena, E., Delon, I., Jones, I., Payre, F., Stern, D.L., 2003. Regulatory evolution of shavenbaby/ovo underlies multiple cases of morphological parallelism. Nature 424, 935-938.

Sucena, E., Stern, D.L., 2000. Divergence of larval morphology between Drosophila sechellia and its sibling species caused by cis-regulatory evolution of ovo/shaven-baby. Proceedings of the National Academy of Sciences of the United States of America 97, 4530-4534.

Thurmond, J., Goodman, J.L., Strelets, V.B., Attrill, H., Gramates, L.S., Marygold, S.J., Matthews, B.B., Millburn, G., Antonazzo, G., Trovisco, V., Kaufman, T.C., Calvi, B.R., 2019. FlyBase 2.0: the next generation. Nucleic acids research 47, D759-D765.

Tomoyasu, Y., 2017. Ultrabithorax and the evolution of insect forewing/hindwing differentiation. Current opinion in insect science 19, 8-15.

Weatherbee, S.D., Halder, G., Kim, J., Hudson, A., Carroll, S., 1998. Ultrabithorax regulates genes at several levels of the wing-patterning hierarchy to shape the development of the Drosophila haltere. Genes \& development 12, 1474-1482.

White, R.A., Wilcox, M., 1984. Protein products of the bithorax complex in Drosophila. Cell 39, 163-171.

White, R.A., Wilcox, M., 1985. Distribution of Ultrabithorax proteins in Drosophila. The EMBO journal 4, 2035-2043.

White, R.A.H., Akam, M.E., 1985. Contrabithorax mutations cause inappropriate expression of Ultrabithorax products in Drosophila. Nature 318, 567-569.

Yuva-Aydemir, Y., Xu, X.L., Aydemir, O., Gascon, E., Sayin, S., Zhou, W., Hong, Y., Gao, F.B., 2015. Downregulation of the Host Gene jigr1 by miR-92 Is Essential for Neuroblast Self-Renewal in Drosophila. PLoS genetics 11, e1005264. 